Trinity University

Digital Commons @ Trinity

Physics and Astronomy Faculty Research

Physics and Astronomy Department

8-2007

\title{
Three-Body Recombination in One Dimension
}

Nirav P. Mehta

Trinity University, nmehta@trinity.edu

B D. Esry

Chris H. Greene

Follow this and additional works at: https://digitalcommons.trinity.edu/physics_faculty

Part of the Physics Commons

\section{Repository Citation}

Mehta, N.P., Esry, B.D., Greene, C.H. (2007). Three-body recombination in one dimension. Physical Review A, 76(2), 022711. doi: 10.1103/PhysRevA.76.022711.

This Article is brought to you for free and open access by the Physics and Astronomy Department at Digital Commons @ Trinity. It has been accepted for inclusion in Physics and Astronomy Faculty Research by an authorized administrator of Digital Commons @ Trinity. For more information, please contact jcostanz@trinity.edu. 


\title{
Three-body recombination in one dimension
}

\author{
N. P. Mehta, ${ }^{1, *}$ B. D. Esry, ${ }^{2, \dagger}$ and Chris H. Greene ${ }^{3, *}$ \\ ${ }^{1}$ JILA, University of Colorado, Boulder, Colorado 80309-0440, USA \\ ${ }^{2}$ Department of Physics, Kansas State University, Manhattan, Kansas 66506, USA \\ ${ }^{3}$ Department of Physics and JILA, University of Colorado, Boulder, Colorado 80309-0440, USA
}

(Received 14 March 2007; published 22 August 2007)

\begin{abstract}
We study the three-body problem in one dimension for both zero- and finite-range interactions using the adiabatic hyperspherical approach. Particular emphasis is placed on the threshold laws for recombination, which are derived for all combinations of the parity and exchange symmetries. For bosons, we provide a numerical demonstration of several universal features that appear in the three-body system, and discuss how certain universal features in three dimensions are different in one dimension. We show that the probability for inelastic processes vanishes as the range of the pairwise interaction is taken to zero and demonstrate numerically that the recombination threshold law manifests itself for large scattering length.
\end{abstract}

DOI: $10.1103 /$ PhysRevA.76.022711

PACS number(s): 34.80.Lx, 34.80.Dp, 34.80.My

\section{INTRODUCTION}

One-dimensional (1D) few-body and many-body systems have been the subject of intense theoretical study for many years [1-6]. This is largely because certain one-dimensional problems admit exact solutions using the Bethe ansatz. These theoretical studies are gaining increasing attention due to the experimental realization of effective $1 \mathrm{D}$ geometries in tightly confined cylindrical trap geometries [7-12]. Atoms in such traps are essentially free to propagate in one coordinate while being restricted to the lowest cylindrical mode in the transverse radial coordinate. Olshanii has shown [13] how the 1D scattering length (which we call $a$ ) is related to the threedimensional $s$-wave scattering length $a_{3 \mathrm{D}}$ and to the oscillator length $a_{\perp}$ in the confined radial direction. This identification allows a connection with the intensively studied 1D zero-range model with two-body interactions

$$
V(x)=g \delta(x) .
$$

The coupling constant $g$ is renormalized to account for virtual transitions to excited radial modes which appear as closed channels in the unrestricted coordinate $x$. Early analytic calculations by McGuire [1] using the interaction Eq. (1) gave vanishing probability for all inelastic events such as collision-induced breakup $(A A+A \rightarrow A+A+A)$ and threebody recombination $(A+A+A \rightarrow A A+A)$. Section IV B discusses how finite-range interactions break the integrability of zero-range models, and also how the probability for recombination and breakup behave with respect to the range of the interaction. In Sec. VI, we comment briefly on the relevance of our 1D model to physical systems in actual atomic waveguides.

In three dimensions (3D), universal features occur when the scattering length is the largest length scale (see [14] and references therein). Adiabatic hyperspherical studies in three dimensions have provided a great deal of insight into such

\footnotetext{
*mehtan@jilau1.colorado.edu

†esry@phys.ksu.edu

${ }^{\ddagger}$ chris.greene@colorado.edu
}

universal behavior [15-18]. For example, the Efimov effect in $3 \mathrm{D}$ appears as a hyperradial potential curve in the region $r_{0} \ll R \ll|a|$ that is attractive and varies as $R^{-2}$. Since it has a supercritical coefficient, this potential has (as $a_{3 \mathrm{D}} \rightarrow \infty$ ) an infinity of long-range bound states spaced geometrically by a universal constant [19]. One of our goals here is to determine what kind of universal behavior, if any, appears in one dimension. We discuss these issues in Sec. V.

A major portion of this work deals with the threshold laws for recombination in 1D. We outline how these laws can be extracted from the asymptotic form of the adiabatic potential curves with a generalized Wigner analysis [20-22], and show that - as with any such analysis - they are independent of the short-range properties of the interactions. This work closely parallels the 3D analysis we carried out in Ref. [22]. Threshold laws are found for all combinations of the parity and exchange symmetries, including the cases where only two of the three particles are identical. In order to demonstrate the threshold behavior, we present numerical calculations for bosons.

Finally, three-body recombination in cold atomic systems results in a measurable loss of trapped atoms. Our goal is to detail the qualitative features of three-body recombination in one dimension in the hopes that such features will be seen in atomic waveguides through atom-loss measurements. Indeed, we provide in Sec. VI, a possible qualitative explanation for the suppression in the three-body loss-rate constant $K_{3}$ measured in Ref. [8]. We speculate that such a measured suppression of atom loss could be connected to both the unique brand of universality discussed in Sec. V, and the suppression predicted by the threshold law in Sec. III. We hope that this theoretical work will stimulate other experiments in this fascinating area of low-dimensional few-body physics.

\section{HYPERSPHERICAL COORDINATES}

Since hyperspherical coordinates and the adiabatic hyperspherical representation play a central role in this paper, but may be unfamiliar to some readers, we will briefly discuss 
the important points. For more detailed information, we refer the interested reader to Refs. [16,18,23].

We separate the center-of-mass motion from the relative motion using Jacobi coordinates,

$$
x_{12}=x_{1}-x_{2} \quad \text { and } \quad x_{12,3}=\frac{m_{1} x_{1}+m_{2} x_{2}}{m_{1}+m_{2}}-x_{3} .
$$

The positions $x_{i}$ locate each particle relative to some laboratory-fixed origin, and $m_{i}$ are their masses. The Jacobi coordinates $x_{12}$ and $x_{12,3}$ constitute a Cartesian coordinate system. Transforming these to polar coordinates $(R, \phi)$,

$$
\mu R^{2}=\mu_{12} x_{12}^{2}+\mu_{12,3} x_{12,3}^{2} \quad \text { and } \quad \tan \phi=\sqrt{\frac{\mu_{12,3}}{\mu_{12}}} \frac{x_{12,3}}{x_{12}}
$$

gives the hyperspherical coordinate system. The reduced masses are

$$
\mu_{12}=\frac{m_{1} m_{2}}{m_{1}+m_{2}} \quad \text { and } \quad \mu_{12,3}=\frac{\left(m_{1}+m_{2}\right) m_{3}}{m_{1}+m_{2}+m_{3}},
$$

and we choose the three-body reduced mass $\mu$ to be $\sqrt{\mu_{12} \mu_{12,3}}$.

With the above definitions, we can find the interparticle distances to be

$$
\left|x_{i}-x_{j}\right|=d_{i j} R\left|\sin \left(\phi-\phi_{i j}\right)\right|,
$$

where we have introduced the constants

$$
\begin{gathered}
\frac{d_{12}}{\sqrt{\mu}}=\sqrt{\frac{1}{\mu_{12}}}, \\
\frac{d_{23}}{\sqrt{\mu}}=\sqrt{\frac{1}{\mu_{12,3}}+\left(\frac{m_{1}}{m_{12}}\right)^{2} \frac{1}{\mu_{12}}}, \\
\frac{d_{31}}{\sqrt{\mu}}=\sqrt{\frac{1}{\mu_{12,3}}+\left(\frac{m_{2}}{m_{12}}\right)^{2} \frac{1}{\mu_{12}}} .
\end{gathered}
$$

The coalescence points-where the interparticle distances are zero-are defined as

$$
\begin{gathered}
\phi_{12}=\frac{\pi}{2}, \\
\tan \phi_{23}=\frac{m_{1}}{m_{12}} \sqrt{\frac{\mu_{12,3}}{\mu_{12}}}, \\
\tan \phi_{31}=\frac{m_{2}}{m_{12}} \sqrt{\frac{\mu_{12,3}}{\mu_{12}}} .
\end{gathered}
$$

If all three particles have the same mass, then $d_{i j}=\sqrt{2 / \sqrt{3}}$ and $\phi_{23}=-\phi_{31}=\pi / 6$. If there are only two identical particles, then it is convenient to label them 1 and 2. In this case, $d_{23}=d_{31}$ and $\phi_{23}=-\phi_{31}$.

Since the hyperradius $R$ is the only length scale in the system, giving the overall size of the system, it is natural to treat it as a "slow" coordinate for an adiabatic representation
[24]. The Hamiltonian for the system can be written as

$$
H=-\frac{1}{2 \mu R} \frac{\partial}{\partial R}\left(R \frac{\partial}{\partial R}\right)+H_{\mathrm{ad}}(R, \phi)
$$

where

$$
H_{\mathrm{ad}}(R, \phi)=-\frac{1}{2 \mu R^{2}} \frac{\partial^{2}}{\partial \phi^{2}}+V(R, \phi)
$$

and $V$ includes the sum of all pairwise interactions. Atomic units have been used here and will be used throughout this work. The adiabatic representation is then defined by the equation

$$
H_{\mathrm{ad}}(R, \phi) \Phi_{\nu}(R ; \phi)=U_{\nu}(R) \Phi_{\nu}(R ; \phi) .
$$

The eigenfunctions $\Phi_{\nu}(R ; \phi)$ are the channel functions, and the eigenvalues $U_{\nu}(R)$ the potential curves corresponding to each channel.

In the limit $R \rightarrow \infty$, the potentials approach either the energies of the bound diatomic molecules for the recombination (two-body) channels, or zero energy for the three-body continuum channels. In this limit, the channels are uncoupled, although there must be coupling at smaller $R$ for any inelastic transition such as recombination to occur. For the purposes of determining the threshold laws, however, we need only know that the channels in this representation become uncoupled asymptotically.

\section{THRESHOLD BEHAVIOR}

At ultracold temperatures, the dominant character of a given scattering process is controlled by its threshold behavior, and the adiabatic hyperspherical picture readily yields this behavior [22]. We show this by first solving the adiabatic equation Eq. (10) while taking into account the appropriate exchange symmetry and parity. In the limit $R \rightarrow \infty$, all of the adiabatic potentials take the general form $U_{\kappa} \rightarrow \kappa^{2} /\left(2 \mu R^{2}\right)$, yielding the hyperradial equation

$$
\left[-\frac{1}{R} \frac{\partial}{\partial R}\left(R \frac{\partial}{\partial R}\right)+\frac{\kappa^{2}}{R^{2}}-k^{2}\right] F(R)=0,
$$

where $k$ is related to the total energy $E(E=0$ at the threebody breakup threshold) by $k^{2}=2 \mu E$. The final momentum in the two-body channel is related to the total energy by $k_{f}^{2}$ $=2 \mu\left(E+B_{2}\right)$, where $B_{2}$ is the two-body binding energy. This equation is simply Bessel's equation with general solution

$$
F_{\kappa}(k R)=A J_{\kappa}(k R)+B Y_{\kappa}(k R) \text {. }
$$

The coefficients $A$ and $B$ are determined by the usual procedure of matching to short-range solutions and are related to the $S$ matrix. It is more convenient for the present discussion, though, to consider each $S$-matrix element for recombination at a fixed total energy $E$ in the form

$$
S_{f i}(E) \sim\left\langle F_{2 B} \Phi_{2 B}|\hat{S}| F_{3 B} \Phi_{3 B}^{\kappa}\right\rangle,
$$

where $2 B$ labels the final atom-dimer channel and $3 B$ labels the initial three-body continuum state. To determine the threshold behavior, we use the small-argument form of the 
Bessel functions. For recombination, the final momentum in the two-body channel is nonzero and slowly varying at the three-body threshold, but the initial momentum $k$ vanishes there. Hence, it is the initial channel that determines the energy dependence of the transition probability, and it is the lowest three-body continuum channel, with $\kappa=\kappa_{\min }$, that will dominate at threshold. As a result, in the limit $k \rightarrow 0$, the recombination probability must scale like

$$
\left|S_{f i}\right|^{2} \propto k^{2 \kappa_{\min }} .
$$

With this scaling, we can, of course, determine the scaling behavior of the recombination rate. This connection will be discussed in Sec. IV A. Moreover, when the scattering length is the largest length scale, simple dimensional arguments (namely, that a probability must be unitless), imply that

$$
\left|S_{f i}\right|^{2} \propto(k a)^{2 \kappa_{\min }} .
$$

\section{A. Three identical bosons with $\delta$-function interactions}

In this section, we will use $\delta$-function pair potentials, Eq. (1), to find $\kappa_{\min }$ for three identical bosons. Even though the recombination rate for such interactions actually vanishes [1], the analytic solutions of the adiabatic equation possible with these interactions [25-28] nevertheless give $\kappa_{\min }$ for general interactions. With $\delta$-function interactions, we can treat only interacting bosons. The case of interacting fermions will be considered in Sec. III B.

For three identical particles, the coalescence points (e.g., $x_{i}-x_{j}=0$ ) form radial lines that are equally spaced by $\pi / 3$ in the two-dimensional space spanned by the Jacobi coordinates defined in Eq. (2). These lines divide the coordinate space into six regions, each of which corresponds to a unique ordering of the three particles along the real line. Symmetry thus permits us to solve the adiabatic equation (10) in just the region $0 \leqslant \phi \leqslant \pi / 6$ with appropriate boundary conditions (see Appendix A).

The $\delta$-function coupling constant $g$ is related to the 1D two-body scattering length by $a=-1 /\left(\mu_{2 B} g\right)\left(\mu_{2 B}=m / 2\right.$ is the two-body reduced mass), which is defined from the 1D effective range expansion in the even-parity "partial wave," $k \tan (\delta)=1 / a+\left(r_{0} / 2\right) k^{2}+\ldots[13,29]$. We require $g<0$ so that the potential supports a two-body bound state, and write the general solution for this channel as

$$
\Phi(R ; \phi)=A \sinh q \phi+B \cosh q \phi
$$

where $q$ is related to the potential energy through

$$
U(R)=-\frac{q^{2}}{2 \mu R^{2}} .
$$

For even parity, we impose boundary conditions $\Phi^{\prime}(0)=0$ and

$$
\lim _{\epsilon \rightarrow 0^{+}} \Phi^{\prime}\left(\phi_{0}+\epsilon\right)-\Phi^{\prime}\left(\phi_{0}-\epsilon\right)=2 \mu g \alpha R \Phi\left(\phi_{0}\right),
$$

where $\alpha=1 / d_{12}=3^{1 / 4} 2^{-1 / 2}$ and $\phi_{0}=\pi / 6$. We require the additional symmetry condition that $\Phi^{\prime}(\pi / 6+\epsilon)=-\Phi^{\prime}(\pi / 6-\epsilon)$. These conditions lead to a transcendental equation for $q$ :

$$
q \tanh \frac{q \pi}{6}=-\mu g \alpha R .
$$

A similar analysis for the continuum solutions begins with the general solution

$$
\Phi(R ; \phi)=A \sin (\kappa \phi)+B \cos (\kappa \phi),
$$

where $\kappa$ is related to the potential energy through

$$
U(R)=\frac{\kappa^{2}}{2 \mu R^{2}},
$$

and is the solution to the following transcendental equation:

$$
\kappa \tan \frac{\kappa \pi}{6}=\mu g \alpha R .
$$

As $R \rightarrow \infty$, the allowed solutions are $\kappa=3,9,15 \ldots$ Note that Eq. (19) is, of course, the analytic continuation of Eq. (22) with $\kappa \rightarrow i q$.

For odd-parity solutions, the only difference is that the boundary condition at $\phi=0$ changes to $\Phi(0)=0$, immediately giving $B=0$ and leading to

$$
q \operatorname{coth} \frac{q \pi}{6}=-\mu g \alpha R
$$

and

$$
\kappa \cot \frac{\kappa \pi}{6}=\mu g \alpha R .
$$

From Eq. (24), the allowed values $\kappa$ for odd-parity bosons asymptotically are $\kappa=6,12,18 \ldots$. Note that the even- and odd-parity potential curves for the two-body channel become degenerate in the limit $R \rightarrow \infty$ as one would expect.

\section{B. Three identical particles with square-well interactions}

In order to determine whether the $\delta$-function results of Sec. III A are general, we now consider square-well pairwise interactions:

$$
V\left(x_{i j}\right)= \begin{cases}-V_{0} & \text { if } 0 \leqslant\left|x_{i j}\right| \leqslant L, \\ 0 & \text { otherwise. }\end{cases}
$$

Like the $\delta$-function interactions, the adiabatic equation remains analytically solvable for square-well interactions. In addition, square-well interactions are a good model for short(but nonzero-) range interactions. At the end of this section, we will point out that the results are, in fact, general for all short-range interactions. Unlike the $\delta$-function interactions, though, indistinguishable fermions can interact via squarewell interactions. Consequently, we will be able to find $\kappa_{\text {min }}$ for three identical fermions.

We thus focus on solutions that are either completely symmetric or completely antisymmetric-assuming as in Sec. III A that the particles are spin polarized such that the spatial wave function carries all of the permutation symmetry. As in Sec. III A, symmetry permits us to to solve the adiabatic equation (10) in a wedge of width $\pi / 6$. To simplify the imposition of boundary conditions, though, we choose 
the interval $\pi / 2 \leqslant \phi \leqslant 2 \pi / 3$ so that the edge $\phi_{b}$ of the square-well centered at $\phi=\pi / 2$ is simply

$$
\cos \phi_{b}=-\left(\frac{3}{4}\right)^{1 / 4} \frac{L}{R}
$$

This condition is invalid at small $R$ where the two-body interactions overlap, but is sufficient for determining the allowed $\kappa$ in the region $R \gg L$. Again using $U(R)=\kappa^{2} /\left(2 \mu R^{2}\right)$
(21) and defining $\beta^{2}=2 \mu R^{2} V_{0}$, the adiabatic equation becomes

$$
\begin{gathered}
\left(\frac{\partial^{2}}{\partial \phi^{2}}+\beta^{2}\right) \Phi=-\kappa^{2} \Phi, \quad \frac{\pi}{2} \leqslant \phi \leqslant \phi_{b}, \\
\frac{\partial^{2}}{\partial \phi^{2}} \Phi=-\kappa^{2} \Phi, \quad \phi_{b} \leqslant \phi \leqslant \frac{2 \pi}{3} .
\end{gathered}
$$

We write the continuum solutions as

$$
\Phi= \begin{cases}A \sin \left[\sqrt{\kappa^{2}+\beta^{2}}(\phi-\pi / 2)\right]+B \cos \left[\sqrt{\kappa^{2}+\beta^{2}}(\phi-\pi / 2)\right], & \pi / 2 \leqslant \phi \leqslant \phi_{b} \\ C \sin [\kappa(\phi-2 \pi / 3)]+D \cos [\kappa(\phi-2 \pi / 3)], & \phi_{b} \leqslant \phi \leqslant 2 \pi / 3\end{cases}
$$

A similar expression may be written for the two-body channels, but we want to focus on the recombination threshold behavior and thus need only the asymptotic behavior of the three-body channels.

For brevity, we sketch the derivation only for even-parity bosons and summarize the results for all other symmetries in Appendix B. For this symmetry, we impose boundary conditions:

$$
\Phi^{\prime}\left(\frac{\pi}{2}\right)=\Phi^{\prime}\left(\frac{2 \pi}{3}\right)=0
$$

which gives $A=C=0$. Matching the logarithmic derivatives of the wave function at $\phi=\phi_{b}$ yields the quantization condition

$$
\sqrt{\kappa^{2}+\beta^{2}} \tan \left[\sqrt{\kappa^{2}+\beta^{2}}\left(\phi_{b}-\frac{\pi}{2}\right)\right]=\kappa \tan \left[\kappa\left(\phi_{b}-\frac{2 \pi}{3}\right)\right] .
$$

In the limit $R \rightarrow \infty, \phi_{b} \rightarrow \pi / 2, \sqrt{\kappa^{2}+\beta^{2}} \rightarrow \beta$, and

$$
\sqrt{\kappa^{2}+\beta^{2}}\left(\phi_{b}-\frac{\pi}{2}\right) \rightarrow \sqrt{2 \mu V_{0}}\left(\frac{3}{4}\right)^{1 / 4} L \equiv \lambda
$$

So, in this limit, Eq. (29) becomes

$$
\beta \tan \lambda=-\kappa \tan \frac{\kappa \pi}{6}
$$

Since $\beta \propto R$, this equation has the same form as Eq. (22), with the same consequences. In particular, the allowed $\kappa$ are $3,9,15, \ldots$.

This analysis can be easily extended to find all allowed values of $\kappa$ for all symmetries (see Appendix B). The threshold law, however, depends only on $\kappa_{\min }$. Collecting this quantity for each symmetry, we find for bosons that

$$
\kappa_{\min }= \begin{cases}3, & \text { even parity, } \\ 6, & \text { odd parity, }\end{cases}
$$

in agreement with the results from Sec. III A for $\delta$-function interactions. For fermions, we find

$$
\kappa_{\min }= \begin{cases}6, & \text { even parity }, \\ 3, & \text { odd parity }\end{cases}
$$

These fermion $\kappa_{\min }$ are actually the same as one would find by symmetrizing the free-particle solutions [see Eq. (A6)]. The interacting boson $\kappa_{\min }$ above, though, are not the same as the symmetrized free-particle solutions from Eq. (A5). From Eq. (14), we see that recombination at threshold will be dominated by the even-parity symmetry for bosons and by odd parity for fermions. Moreover, recombinations of bosons and fermions [30] in 1D share the same threshold law.

The above analysis, in fact, generalizes to short-range potentials of any form [i.e., $V(x)=0$ for $x \gg L$ ]. That is, if we take the logarithmic derivative $b$ [see Eq. (C3)] from the two-body equation outside the range of the interaction, then the matching condition is

$$
\left(\frac{4}{3}\right)^{1 / 4} b R=\kappa \tan \left[\kappa\left(\phi_{b}-\frac{2 \pi}{3}\right)\right]
$$

for even-parity bosons. In the limit $R \rightarrow \infty, b$ may be regarded as a constant so that this equation reduces to the same form as Eq. (22) or Eq. (31) and carries the same consequences.

\section{Two identical particles with $\delta$-function interactions}

For this case, all combinations of parity and exchange are considered, assuming that the spins of each particle are fixed by spin polarization. Following the discussion in Sec. II, we label the two indistinguishable particles 1 and 2. The interaction then takes the form

$$
V=g_{S} \delta\left(x_{12}\right)+g_{D}\left[\delta\left(x_{31}\right)+\delta\left(x_{23}\right)\right],
$$

where $g_{S}$ denotes the same-particle coupling, $g_{D}$ denotes the different-particle coupling, and $x_{i j}$ are the interparticle distances. The constant $\alpha$ in Eq. (18) is equal to $1 / d_{i j}$ for each 
pair of particles $i j$. We shall use $\alpha_{S}$ for particles 1 and 2 and $\alpha_{D}$ otherwise.

By symmetry, we need only solve the adiabatic equation in the range $0 \leqslant \phi \leqslant \pi / 2$. The $\delta$-function boundary condition
Eq. (18) is imposed at $\phi_{0}=\phi_{23}$ for the distinguishable pair [see Eq. (7)] and at $\phi_{0}=\pi / 2$ for the indistinguishable pair. The general continuum solution is now conveniently written as

$$
\Phi= \begin{cases}A \cos (\kappa \phi)+B \sin (\kappa \phi), & 0 \leqslant \phi \leqslant \phi_{23}, \\ C \cos [\kappa(\phi-\pi / 2)]+D \sin [\kappa(\phi-\pi / 2)], & \phi_{23} \leqslant \phi \leqslant \pi / 2 .\end{cases}
$$

We are now prepared to determine the asymptotically allowed values of $\kappa$ for specific symmetries.

For brevity, we outline the derivation only for even-parity bosons and summarize the results for all cases in Appendix B. As before, even-parity bosons require the boundary conditions

$$
\Phi^{\prime}(0)=0 \quad \text { and } \quad \Phi^{\prime}\left(\frac{\pi}{2}+\epsilon\right)=-\Phi^{\prime}\left(\frac{\pi}{2}-\epsilon\right) .
$$

These conditions immediately give $B=0$ and yield the quantization condition

$$
\begin{aligned}
(-\kappa & \left.+\frac{2}{\kappa} \mu^{2} g^{2} \alpha_{S} \alpha_{D} R^{2}\right) \tan \left[\kappa\left(\phi_{23}-\frac{\pi}{2}\right)\right] \\
& -\mu g \alpha_{S} R \tan \kappa \phi_{23} \tan \left[\kappa\left(\phi_{23}-\frac{\pi}{2}\right)\right]+\kappa \tan \kappa \phi_{23} \\
& =\mu g\left(\alpha_{S}+2 \alpha_{D}\right) R .
\end{aligned}
$$

In the limit $R \rightarrow \infty$, the $R^{2}$ term dominates, and this equation reduces to

$$
\frac{1}{\kappa} \tan \kappa\left(\phi_{23}-\frac{\pi}{2}\right)=0
$$

yielding solutions $\kappa=n \pi /\left|\phi_{23}-\pi / 2\right|$ for $n=1,2,3, \ldots$ Careful inspection of Eq. (37) leads to the additional solution

$$
\kappa \tan \kappa \phi_{23} \rightarrow \infty
$$

This equation has solutions $\kappa=(n+1 / 2) \pi /\left|\phi_{23}\right|$ for $n$ $=0,1,2, \ldots$.

Similar analyses can be carried out for all combinations of parity and exchange (see Appendix B). The resulting $\kappa_{\min }$ for each symmetry are summarized below. As discussed above, $\kappa_{\text {min }}$ determines the threshold law for recombination and is, for two identical particles in general, an irrational number that depends only on the masses. Moreover, since $\kappa_{\min }$ is always a nonzero quantity, we find that the recombination rate is never a constant at threshold in 1D. If we let the two identical particles be labeled 1 and 2 with mass $M$, and the third particle be labeled 3 with mass $m$, for bosons we have

$$
\kappa_{\min }=\left\{\begin{array}{lll}
\left\{\frac{\pi}{\left|\phi_{23}-\pi / 2\right|},\right. & \phi_{23} \leqslant \pi / 6 \quad(M \geqslant m), & \\
\frac{\pi}{2\left|\phi_{23}\right|}, & \phi_{23}>\pi / 6, & (M<m), \\
\frac{\pi}{\left|\phi_{23}-\pi / 2\right|}, & \text { even parity },
\end{array}\right.
$$

while for fermions we have

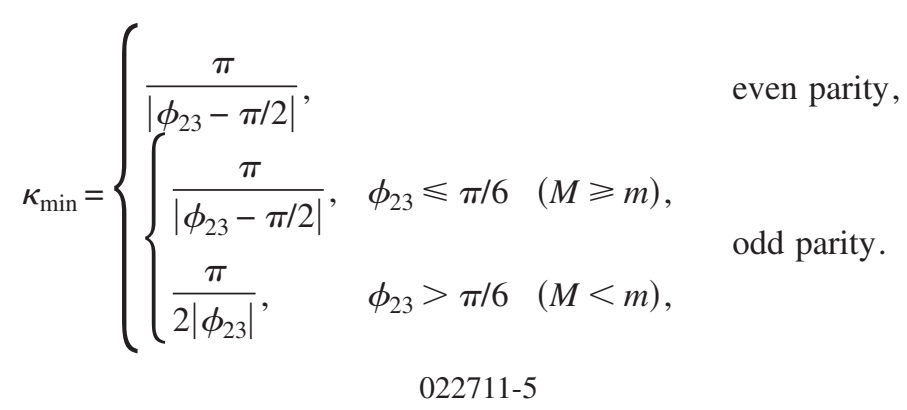


Note that these results do not all immediately reduce to the equal-mass results (i.e., $\phi_{23}=\pi / 6$ ) of Sec. III B since the symmetry at $\phi_{23}$ has not been taken into account here. That symmetrization eliminates some solutions, finally giving complete agreement with the equal-mass results.

\section{RECOMBINATION FOR BOSONS}

\section{A. Cross section and event rate constant}

In the presence of a purely short-range hyperradial potential, the scattering solution for distinguishable particles is of the form

$$
\Psi^{\mathrm{dist}} \rightarrow e^{i k R \cos \left(\phi-\phi^{\prime}\right)}+f^{3 B} \frac{e^{i k R}}{\sqrt{R}},
$$

where the "direction" of the incident plane wave is parametrized by the angle $\phi^{\prime}$. For $\phi^{\prime}=0$, the incident flux is in the direction of the first Jacobi vector $x_{12}$, while for $\phi^{\prime}=\pi / 2$, the incident flux is in the direction of $x_{12,3}$. The quantity $f^{3 B}$ represents the elastic three-body scattering amplitude and is given by

$$
f^{3 B}=\sqrt{\frac{1}{2 \pi k}} \sum_{m=-\infty}^{m=\infty} e^{i m\left(\phi-\phi^{\prime}\right)}\left(e^{2 i \delta_{m}}-1\right) .
$$

We have defined the scattering phase shift in the $m$ partial wave as $\delta_{m}$. The total integrated cross section is then [31]

$$
\sigma_{\mathrm{dist}}=\int_{0}^{2 \pi} d \phi\left|f^{3 B}\right|^{2}=\frac{1}{k} \sum_{m=-\infty}^{m=\infty}\left|e^{2 i \delta_{m}}-1\right|^{2} .
$$

This expression must be modified to account for three separate issues. First, we are interested in the cross section for an inelastic process. Second, we must account for the appropriate identical particle symmetry, and third, our asymptotic harmonics are not two-dimensional partial waves.

Taking these issues into consideration, we findfollowing the arguments of [32] - that the recombination cross section for identical bosons in terms of the $S$ matrix is

$$
\sigma=\frac{6}{k} \sum_{\kappa, p}\left|\left\langle\Phi_{2 B, p}^{\mathrm{sym}}|\hat{S}| \kappa\right\rangle\right|^{2}
$$

where $\Phi_{2 B, p}^{\mathrm{sym}}$ denotes the final symmetrized two-body channel function with overall three-body parity $p$, and $\kappa$ labels the three-body entrance channel. At ultracold temperatures, we need only include the smallest $\kappa=\kappa_{\text {min }}$, giving

$$
\sigma=\frac{6}{k}\left|\left\langle\Phi_{2 B, e}^{\mathrm{sym}}|\hat{S}| \kappa=3\right\rangle\right|^{2} .
$$

Similar expressions can be derived for three identical fermions and for systems with only two identical particles. In fact, the only change for these other symmetries-besides having different dominant $\kappa$ and parity at threshold-is the numerical prefactor.

We are interested not only in the cross section, but also in the more experimentally relevant event rate constant,

$$
K_{3}=\frac{\hbar k}{\mu} \sigma=\frac{6 \hbar}{\mu}\left|S_{2 B, 3 B}^{\mathrm{sym}}\right|^{2} .
$$

Here, we explicitly show all factors of $\hbar$ in order to emphasize that this quantity has the appropriate units of (length) $)^{2}$ (time). $K_{3}$ represents the probability of a recombination event per atomic triad per unit density of atomic triads per unit time. The volume is the full volume spanned by the internal coordinates of the three-particle system, so that the density is the number of triads per unit two-dimensional volume. As defined in Eq. (45), the rate is a function of energy. To compare with experiment, though, it should be thermally averaged to give the rate as a function of temperature. This averaging is especially important in 1D since $K_{3}(E)$ is not constant at threshold.

\section{B. Zero- vs finite-range interactions}

For identical bosons with $\delta$-function interactions, the elastic atom-dimer $S$-matrix element is $[1,4,26]$

$$
S_{11}=\exp (2 i \delta)=1-\frac{24\left(k_{12,3} a\right)}{\frac{9}{2} i\left(k_{12,3} a\right)^{2}+12\left(k_{12,3} a\right)-6 i}
$$

where $k_{12,3}=\sqrt{2 \mu_{12,3}\left(E+B_{2}\right)}$. Simple analysis of Eq. (46) shows that $\left|S_{11}\right|^{2}=1$ for all energies $E>-B_{2}$ where this is an open scattering channel, meaning that scattering in the twobody channel is always elastic. Hence, the amplitude for breakup (and also recombination) is identically zero at all energies.

For $\delta$-function interactions, the adiabatic hyperspherical potential curves have previously been calculated by solving the transcendental equation Eq. (22) [25,26,33]. Since we want to calculate inelastic transition rates, we also require the nonadiabatic couplings $P_{i j}$ and $Q_{i j}$ in Eqs. (C7) and (C8). In general, the preferred way to calculate these couplings numerically is from difference formulas. It is difficult to obtain high accuracy by differencing, though. A numerical algorithm for calculating nonadiabatic couplings to the same accuracy as the adiabatic eigenvalues was given in [34,35]. In cases where the adiabatic solution $\Phi$ can be written analytically, however, it is possible to calculate $\partial \Phi / \partial R$ directly [36]. This is accomplished by differentiating the transcendental equations for $\kappa$ in Sec. III to determine equations for $\partial \kappa / \partial R$. Rather lengthy expressions for $P_{i j}$ and $Q_{i j}$ result, but they do allow the couplings to be calculated essentially exactly. The first few elements of the first row of the antisymmetric matrix $\mathbf{P}$ are shown in Figs. 1(b) and 1(d). The important feature of this figure is simply that these elements are not zero. They couple the two- and three-body channels, yet we know from Eq. (46) that the amplitude for any process connecting the two- and three-body channels must vanish. We will numerically demonstrate that, despite the nonzero couplings, the solution to the coupled radial equations indeed gives vanishing probability for inelastic events.

In order to facilitate this demonstration, we consider the Pöschl-Teller two-body potential 

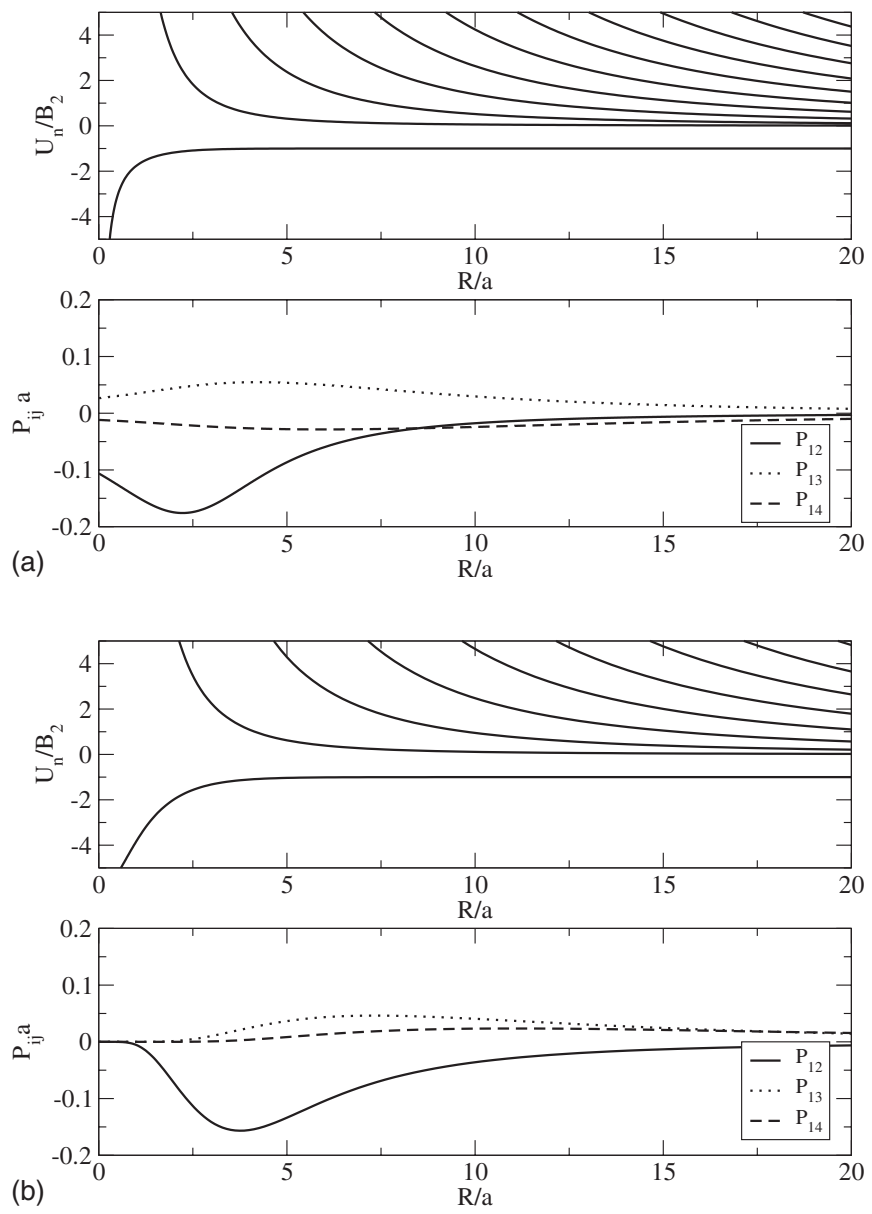

FIG. 1. Adiabatic potential curves (a) and first-derivative couplings (b) for $\delta$-function interactions $(a=2)$; adiabatic potential curves (c) and first derivative couplings (d) for the Pöschl-Teller potential with $L=a=2$. All axes are dimensionless.

$$
V(x)=-D \operatorname{sech}^{2} \frac{x}{L}, \quad D>0,
$$

which gives a Schrödinger equation having an analytic solution [37]. Defining $\eta=\sqrt{1+4 D m L^{2}}$, the zero-energy scattering length for the even-parity solution is

$$
a=\frac{L}{2}\left(H_{-1 / 2-\eta / 2}+H_{-1 / 2+\eta / 2}-\pi \sec \eta \frac{\pi}{2}\right) \text {. }
$$

In this expression, $H_{x}=\gamma_{\mathrm{EM}}+\psi(x+1), \gamma_{\mathrm{EM}}$ is the EulerMascheroni constant, and $\psi(x)=\Gamma^{\prime}(x) / \Gamma(x)$ is the digamma function. The energy eigenvalues are

$$
E=-\frac{(2 n+1-\eta)^{2}}{4 m L^{2}}, \quad n=0,1,2, \ldots
$$

The scattering length becomes infinite when an even-parity state sits at threshold, that is, when $E=0$ for $n=0,2,4, \ldots$.

To check whether recombination with the Pöschl-Teller potential recovers the $\delta$-function result (46), we set $m=1$, fix the scattering length to $a=2$, and consider the effect of letting $L \rightarrow 0$, approaching the $\delta$-function limit. When $L=2$ and $D=\frac{1}{2}$, the Pöschl-Teller potential gives the same two-body

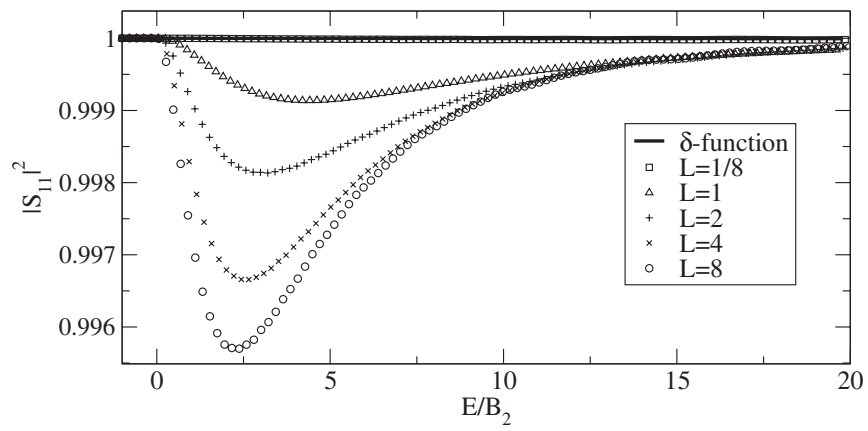

FIG. 2. Convergence of $\left|S_{11}\right|^{2}$ for finite-range potentials (with range $L$ ) to the zero-range theory is shown. The scattering length is held fixed at $a=2$ as $L$ in Eq. (47) is taken to zero. The data for $L=1 / 8$ (squares) are nearly indistinguishable from the solid $\delta$-function line. All axes are dimensionless.

binding energy and scattering length as the $\delta$ function. The only difference is that the former is of finite range. The difference in the resulting three-body potential curves and couplings, however, is more subtle. These potentials and couplings are shown in Figs. 1(c) and 1(d). By comparison with the $\delta$-function results in Figs. 1(a) and 1(b), we see that all features for the Pöschl-Teller results tend to be pushed to larger $R$.

Our numerically obtained $\left|S_{11}\right|^{2}$ for both kinds of potentials are shown in Fig. 2. The numerical techniques used for all calculations in this work are described in Appendix C. Below the dimer breakup threshold at $E=0$, the collision is purely elastic. Above the breakup threshold, the phase shift in the elastic channel acquires an imaginary part, which appears as a deviation from $\left|S_{11}\right|^{2}=1$, implying a nonzero probability for breakup of the dimer. As indicated in the figure, our numerical calculations show that as $L \rightarrow 0$, the collisions do indeed become purely elastic at all energies, in agreement with Eq. (46). Since the $S$ matrix is symmetric under time reversal, the amplitude for recombination also vanishes. It should be stressed that the solid black line in Fig. 2 is the result of our numerical calculation for the $\delta$ function, and not simply a plot of the analytical result Eq. (46).

\section{Low-energy effective interaction}

While the potential Eq. (47) has the advantage of yielding an exact analytic two-body solution, it does not facilitate the study of recombination into a single high-lying two-body state in the limit $a \rightarrow \infty$. The disadvantage stems from the fact that the potential is purely attractive. As is well known, any purely attractive potential in 1D will support an even-parity bound state no matter how small the coupling. Hence, for the potential Eq. (47) to have $a \rightarrow \infty$ with a single bound state, we must let $D \rightarrow 0$. Therefore, we are motivated to construct a renormalized low-energy potential model that supports a single (shallow) bound state with finite couplings in the limit $a \rightarrow \infty$. We follow the insight of Ref. [38] (see also Ref. [29]) and take

$$
V_{\mathrm{eff}}(x)=\frac{\Lambda^{2}}{m}\left[c-2 d+4 d(\Lambda x)^{2}\right] \exp \left(-\Lambda^{2} x^{2}\right) .
$$

The prefactor $\Lambda^{2} / m$ gives the interaction units of energy in atomic units, and $m$ is the mass of the helium atom in atomic 


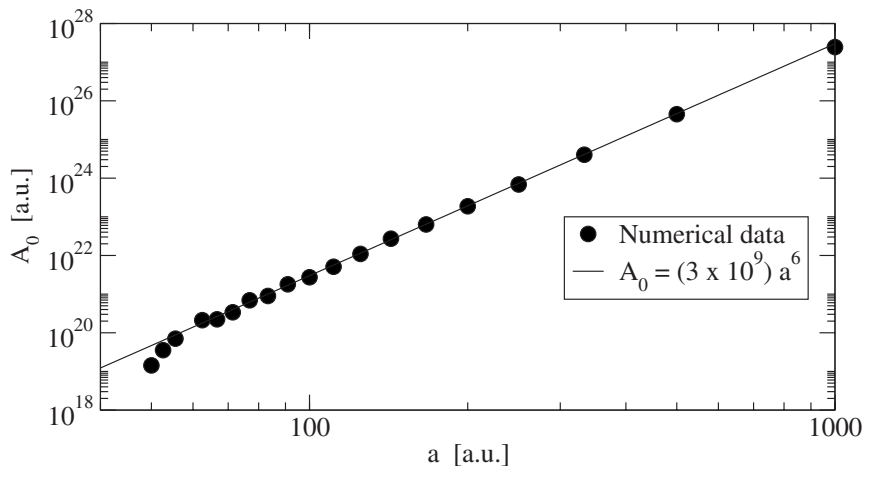

FIG. 3. Numerical demonstration of $a^{6}$ scaling of the three-body recombination probability. The black line is $A_{0}=3.0 \times 10^{9} a^{6}$, and the circles are our numerical results. The units of $A_{0}$ are atomic units of inverse energy cubed, consistent with the use of Eq. (50) to model the ${ }^{4} \mathrm{He}$ two-body interaction.

units $\left(m=7296.299 m_{e}\right)$. One can tune the free couplings $c$ and $d$ so that the potential reproduces physically reasonable values for the scattering length and effective range of, for example, ${ }^{4} \mathrm{He}$ [39]: $a=208$ a.u. and $r_{0}=14$ a.u. A reasonable requirement of any renormalized model is that low-energy observables remain independent of the momentum cutoff $\Lambda$. We find that the values $c=1.97151$ and $d=-1.46165$ for $\Lambda=0.16$ a.u. yield the desired effective range parameters. These values also give a two-body binding $B_{2}=3.39985$ $\times 10^{-9}$ a.u. We have verified that $B_{2}$ varies by less than $1 \%$ over a wide range of cutoffs $0.08 \leqslant \Lambda \leqslant 0.3$, and that the couplings $c$ and $d$ are of order unity over this range.

\section{Low-energy scaling behavior}

From Eq. (14) and Sec. III, we know that the inelastic transition probability must scale as $\left|S_{12}\right|^{2} \sim k^{6} \sim E^{3}$ at threshold since $\kappa_{\min }=3$ for three bosons. This behavior is already plausibly seen in Fig. 2 since this scaling for $S_{12}$ implies 1 $-\left|S_{11}\right|^{2} \sim E^{3}$ at the three-body breakup threshold. When the scattering length $a$ is the longest length scale, we also know from Eq. (15) that $\left|S_{12}\right|^{2} \sim(k a)^{6} \sim E^{3} a^{6}$. We demonstrate this behavior quantitatively in Fig. 3. The points in the figure were obtained by first tuning the effective potential Eq. (50) to reproduce a given scattering length, and then calculating the recombination probability near threshold, $E \rightarrow 0$. Finally, we divided the probability by $E^{3}$ to extract the constant of proportionality. The constant $A_{0}$ in $\left|S_{12}\right|^{2}=A_{0} E^{3}$ is plotted as a function of the scattering length in Fig. 3. The $a^{6}$ scaling is clearly seen when compared to the solid line, which is $A_{0}$ $=3.0 \times 10^{9} a^{6}$.

It is interesting to note that the lack of inelastic processes for zero-range interactions is actually a consequence of perfect destructive interference in the exit channel. Indeed, since the couplings $P_{i j}$ and $Q_{i j}$ are nonzero, the only way for the inelastic probability to vanish is through some sort of interference effect. It is possible to demonstrate this perfect interference by adding an arbitrary short-range three-body interaction $V_{3}(R)$ of characteristic length $L_{3}$ to the zero-range hyperradial potentials. The short-range three-body interaction destroys the perfect interference and leads to a nonzero recombination probability. Considering the ratio $C=(1$ $\left.-\left|S_{11}\right|^{2}\right) /(k a)^{6}$ near threshold, we find a nonuniversal power law (that depends on the short-range nature of $V_{3}$ ) of the form $C \sim\left(L_{3} / a\right)^{c_{1}}$. Although the particular value of $c_{1}$ is nonuniversal, we always find $c_{1}>0$ such that $K_{3} \rightarrow 0$ as $L_{3} \rightarrow 0$.

\section{UNIVERSALITY IN ONE DIMENSION}

In three dimensions, the three-body problem exhibits universal features in the limit $|a| \rightarrow \infty$ related to Efimov physics $[14,16,19,40]$. Here, we consider this limit in one dimension. For $L \ll R \ll|a|$-with $L$ generally the characteristic size of the two-body interaction-we find that

$$
q^{2}=-\kappa^{2} \approx \frac{12 \alpha R}{\sqrt{3} \pi a}
$$

to leading order in $R / a$ for $a>0$ and $a<0$, respectively. The corresponding adiabatic potentials are

$$
U(R)=\frac{\gamma_{0}}{2 \mu a R}
$$

with

$$
\gamma_{0}=-\frac{12}{3^{1 / 4} 2^{1 / 2} \pi} \approx-2.052277
$$

We thus find an attractive, universal $R^{-1}$ potential when $a$ $>0$, and a similarly universal, repulsive $R^{-1}$ potential when $a<0$. In the former case, this universal potential converges to the highest-lying two-body threshold while for the latter, it is the lowest potential converging to the three-body breakup threshold.

This result can be derived in several ways. For instance, the $\delta$-function transcendental equations Eqs. (19) and (22) for positive and negative scattering lengths, respectively, can be solved in the small- $q$ or $-\kappa$ limit. The exact same universal curves can similarly be extracted from the analogous quantization equations for the square-well interaction [Eq. (29), for example] using

$$
\beta=\left(\frac{4}{3}\right)^{1 / 4} \frac{R}{L} n \pi\left(1+\frac{L}{n^{2} \pi^{2} a}\right), \quad n>0,
$$

assuming there are $n$ two-boson bound states. This choice for $\beta$ follows from the fact that the two-body square well has a zero-energy bound state when $\sqrt{2 \mu_{2 B} V_{0}} L=n \pi$. Reducing this phase slightly gives $a<0$; and increasing it gives $a>0$. Finally, the universal curves can be obtained quite generally from the quantization conditions for arbitrary short-range potentials, Eq. (33), using this expression for the logarithmic derivative in the limit $2 \mu_{2 B} E L^{2} \ll 1$ :

$$
b=\frac{1}{a-L} .
$$

It is clear that the universality of the $1 \mathrm{D}$ problem has a different character from that of the $3 \mathrm{D}$ problem. First, $\gamma_{0}$ is the coefficient of $R^{-1}$ instead of $R^{-2}$ as in 3D. Second, the universal potential Eq. (52) actually vanishes in the limit $|a|$ 


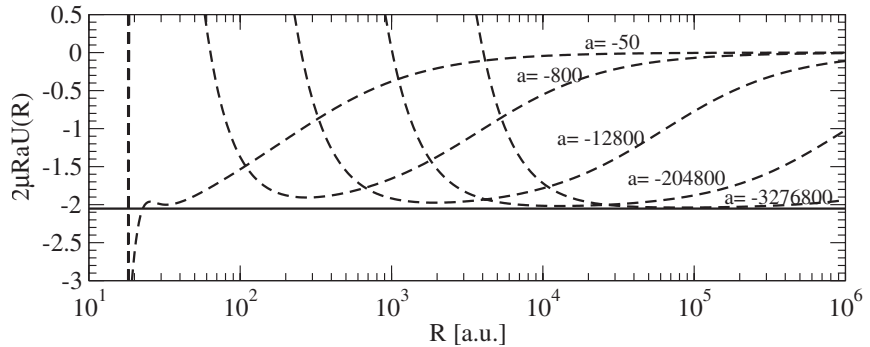

FIG. 4. Lowest adiabatic hyperspherical potential curve for the two-body interaction in Eq. (50) with several different values of $a$ $<0$ ( $a$ given in a.u.). The curve is multiplied by the factor $2 \mu R a$ to give a unitless quantity and more clearly reveal where the potential behaves as a repulsive $R^{-1}$. The horizontal solid line is the universal number $\gamma_{0} \approx-2.05(53)$.

$\rightarrow \infty$. The lowest three-body continuum potential in this case is instead an attractive $R^{-3}$ potential. For square-well interactions, we can derive it explicitly using $\beta$ from Eq. (54) with $|a| \rightarrow \infty$ in Eq. (29), yielding

$$
U(R)=-\left(\frac{3}{4}\right)^{1 / 4} \frac{n^{2} \pi L}{2 \mu R^{3}} .
$$

This potential, however, is not universal, although numerical calculations with four different short-range two-body potentials suggest that two aspects are general: (i) the attractive $R^{-3}$ behavior and (ii) the increasing interaction strength with $n$.

As $|a| \rightarrow \infty$, the solution in the lowest channel in the region $L \ll R \ll|a|$ will obey the equation

$$
\left[-\frac{1}{2 \mu R} \frac{\partial}{\partial R}\left(R \frac{\partial}{\partial R}\right)+\frac{\gamma_{0}}{2 \mu a R}-\frac{Q_{11}(R)}{2 \mu}\right] F(R)=E F(R) .
$$

The diagonal nonadiabatic coupling $-Q_{11}(R)$ is always repulsive and falls off as $R^{-2}$, while the universal $R^{-1}$ term will vary smoothly from an attractive to a repulsive potential as $a$ varies from $+\infty$ to $-\infty$. When $|a|=\infty$, of course, the $R^{-1}$ potential vanishes, leaving just the $R^{-3}$ potential in Eq. (56).

In order to demonstrate that $\gamma_{0}$ is indeed a universal constant, we again turn to the effective potential Eq. (50). We have calculated three-body potential curves using the twobody potential Eq. (50) tuned to give different two-body scattering lengths (the effective range is held constant at 14 a.u.). Figure 4 shows the lowest three-body potential for increasingly negative scattering lengths. The potential curves have been multiplied by the factor $2 \mu a R$ in order to more clearly reveal the universal behavior. We see that the curves do in fact approach $\gamma_{0}$ over a range of $R$ consistent with the condition $r_{0} \ll R \ll|a|$. At $R \gg|a|$, the potentials again approach the three-body breakup threshold with the $R^{-2}$ behavior predicted in Sec. III (which translates to $R^{-1}$ as plotted in the figure).

For large positive scattering lengths, the lowest potential curve - the two-body channel-supports a three-body bound state so long as there is at most one weakly bound two-body state. Figure 5 shows the lowest potential curve for various

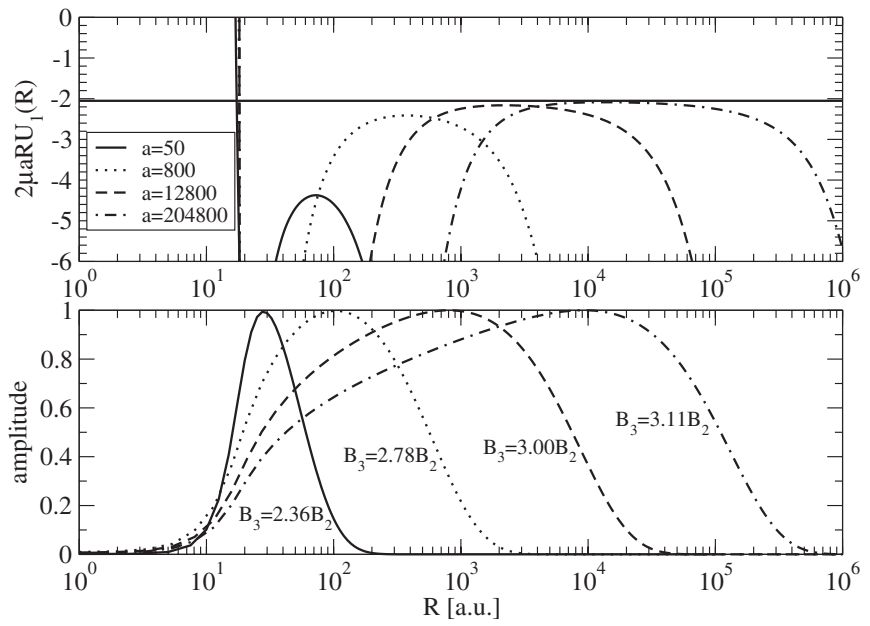

FIG. 5. (a) Lowest adiabatic hyperspherical potential curve for the two-body interaction in Eq. (50) with several different values of $a>0$ (units of $a$ are a.u.). The curve is multiplied by the factor $2 \mu R a$ to reveal the region in which the potential behaves as an attractive $R^{-1}$. The horizontal solid line is the universal number $\gamma_{0} \approx-2.05$ (53). (b) Hyperradial wave functions for each potential in (a). The wave functions have been scaled such that their maximum is unity. As $a \rightarrow \infty$, we expect the three-body binding $B_{3}$ $\rightarrow 4 B_{2}$, as is the case for $\delta$-function interactions [1].

values of the two-body scattering length along with the hyperradial wave functions for the three-body bound state in this channel. It is clear that the universal $R^{-1}$ portion of the curve has a strong influence on these states since a significant portion of the necessary phase is accumulated in this region. This argument is supported by a simple Wentzel-KramersBrillouin (WKB) calculation using the Bohr-Sommerfeld quantization condition

$$
\int_{R_{1}}^{R_{2}} d R \sqrt{2 \mu E-\frac{\gamma_{0}}{a R}}=\left(n+\frac{1}{2}\right) \pi
$$

for classical turning points $R_{i}$. We note that the Langer correction does not appear in this equation since it cancels the attractive $R^{-2}$ term obtained from eliminating the first derivative in the hyperradial kinetic energy-which is required to use this WKB phase integral. The energies of the nodeless solutions as $a \rightarrow \infty$ using the above equation are in reasonable agreement with a $B$-spline calculation using the numerical potential curves. These results are tabulated in Table I.

TABLE I. The three-body bound-state energies are shown in atomic units for various two-body potentials of the form Eq. (50) tuned to give the scattering lengths in the first column. The WKB estimates improve as $a \rightarrow \infty$.

\begin{tabular}{lcc}
\hline \hline$a$ & Numerical $B$ spline & WKB \\
\hline 50 & $-1.8644 \times 10^{-7}$ & $-4.8391 \times 10^{-8}$ \\
800 & $-6.0569 \times 10^{-10}$ & $-4.8839 \times 10^{-10}$ \\
12800 & $-2.5156 \times 10^{-12}$ & $-2.6829 \times 10^{-12}$ \\
204800 & $-1.0324 \times 10^{-14}$ & $-1.1533 \times 10^{-14}$ \\
\hline \hline
\end{tabular}




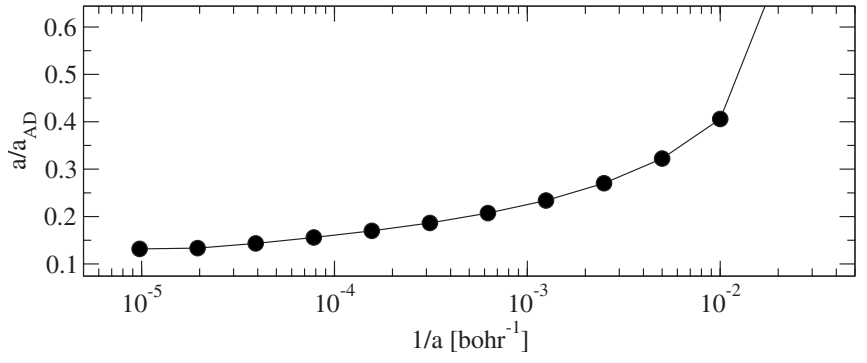

FIG. 6. Inverse of the atom-dimer scattering length multiplied by the atom-atom scattering length as a function of the inverse of the atom-atom scattering length. In the limit that $a \rightarrow \infty$, we find approximately $a_{\mathrm{AD}} / a \rightarrow 8.5$.

Further examination of Eq. (58) also shows why-despite the long-range $R^{-1}$ behavior-there is only a single threebody bound state. If we evaluate the WKB phase at the twobody threshold for $R$ between $L$ and $a$, then we find that it lies between $\pi / 2$ and $0.817 \pi$ for all $a / L$ between 8.07286 and infinity. The phase remains finite because the strength of the $R^{-1}$ potential decreases with increasing $a$ at the same time that the domain over which it holds grows with $a$. So there is sufficient phase accumulated in this universal region alone to support a single bound state by Eq. (58). Moreover, the phase contributed from the small- $R$ region, $R \leqslant L$, would have to exceed roughly $0.7 \pi$ to produce a second bound state. While this is not impossible, it does not seem likely.

Finally, we consider the atom-dimer scattering length $a_{\mathrm{AD}}$ in the universal limit. Again using Eq. (50), we calculate $a_{\mathrm{AD}}$ as $a \rightarrow \infty$. Our results (plotted in Fig. 6) show a clear trend toward $a_{\mathrm{AD}} \approx 8.5 a$. This finding is consistent with the presence of a bound state at the atom-dimer threshold in the $\delta$-function model $[1,26,33]$. As $a \rightarrow \infty$ this zero-energy resonance results in $a_{\mathrm{AD}} \rightarrow \infty$.

Before we end our discussion of universality, it is worth mentioning the consequences for the recombination rate. So long as $a$ is finite, the lowest three-body continuum potential still behaves as predicted in Sec. III for $R \gg|a|$. The powerlaw scaling of the $S$ matrix with $E$ and $a$ is thus the same as found in that section. The $a$ dependence is modified, though, by the universal region of the potential. Using arguments similar to those in Refs. $[17,41]$, we can use a WKB calculation to determine the modifications. For $a<0$, the coupling driving recombination peaks around $R=L$, so the system must tunnel through the repulsive $R^{-2}$ potential at $R \gg|a|$ and through the repulsive $R^{-1}$ universal in the region $L \ll R \ll|a|$ in order to recombine. The WKB tunneling integral, $\int_{L}^{a} d R \sqrt{\gamma_{0} /|a| R}$, then leads to the modified threshold scaling for $a<0$

$$
K_{3} \propto \frac{\hbar}{\mu}(k a)^{6} \exp \left[-4 \sqrt{\left|\gamma_{0}\right|}\left(1-\xi \sqrt{\frac{L}{|a|}}\right)\right]
$$

where $\xi$ is a numerical constant on the order of unity that depends on the exact range of $R$ over which the universal potential is valid. Equation (59) does not go zero when $L$ $\rightarrow 0$ as one might expect, but it does take on its minimum value. This is simply because the WKB estimate does not capture the peculiar interference required to make $K_{3}$ exactly zero in the limit $L \rightarrow 0$.

For $a>0$, in analogy to the 3D case, recombination can be modified by interference to give

$$
K_{3} \propto \frac{\hbar}{\mu}(k a)^{6} \sin ^{2}\left[2 \sqrt{\left|\gamma_{0}\right|}\left(1-\xi \sqrt{\frac{L}{|a|}}\right)+\Phi\right]
$$

where $\Phi$ is the short-range $R \ll L$ phase accumulated in the nonuniversal portion of the two-body channel.

When $|a|=\infty$ and the incident three-body continuum potential is given by Eq. (56), the threshold scaling is nontrivially modified. The attractive $R^{-3}$ potential is equivalent to $\kappa_{\min }=0$, so the recombination rate is, in fact, independent of energy at threshold, $K_{3} \propto$ const. It turns out that odd-parity, identical fermions share this threshold law in the limit that the two-body odd-parity partial wave scattering lengthappropriate for fermion-fermion interactions-goes to infinity. The threshold scaling for odd-parity bosons and evenparity fermions is also changed from the predictions of Sec. III. In the limit that their respective scattering lengths are infinite, $K_{3} \propto E^{3}$ for both cases.

\section{DISCUSSION AND SUMMARY}

The work presented here has been carried out in strictly one dimension. It is worth commenting on the relation of this study to experimentally realizable, effective 1D geometries. Olshanii [13] has determined the effective 1D scattering amplitude for two particles with 3D $s$-wave scattering length $a_{3 \mathrm{D}}$ interacting under strong cylindrical harmonic confinement. His analysis leads to the following one-dimensional effective range expansion:

$$
k \tan \delta=\frac{1}{a}+\frac{\zeta(3 / 2) a_{\perp}^{3}}{8 a^{2}} \frac{1}{2} k^{2}+O\left(k^{4}\right),
$$

where $a_{\perp}$ is the oscillator length in the confined direction, and $\zeta(x)$ is the Riemann zeta function. The 1D scattering length is thus determined at zeroth order in $k$ to be

$$
a=-\frac{a_{\perp}^{2}}{2 a_{3 D}}\left(1+\zeta(1 / 2) \frac{a_{3 D}}{a_{\perp}}\right) .
$$

One may argue that the 3D effective range should also be present in the $k^{2}$ term of Eq. (61), but since $a_{\perp} \gg r_{0}^{3 \mathrm{D}}$ in general, it is reasonable to assume that the contribution from $r_{0}^{3 \mathrm{D}}$ is small compared to $a_{\perp}$. With this approximation in hand, it is possible to calculate the 3D parameters $a_{\perp}$ and $a_{3 \mathrm{D}}$ that correspond to the 1D parameters $a$ and $r_{0}$ that we have used. For $r_{0}=14$ a.u. and our larger values of $a$, we find experimentally accessible values for $a_{\perp}$ and $a_{3 \mathrm{D}}$. All values are tabulated in Table II. One notable point is that the $3 \mathrm{D}$ scattering length is negative for positive 1D scattering lengths. So while there is no shallow two-body bound-state in the $3 \mathrm{D}$ system, a shallow bound state appears as a result of the cylindrical confinement.

Finally, we comment on the role of three-body recombination in one recent experiment. The experiment by Tolra et al. [8] has measured three-body recombination rates in 
TABLE II. The 3D parameters $a_{3 D}$ and $a_{\perp}$ are calculated to give the desired $1 \mathrm{D}$ effective range $r_{0}=14$ a.u. and the $1 \mathrm{D}$ scattering length in the first column.

\begin{tabular}{lcr}
\hline \hline$a$ (a.u.) & $a_{3 D}$ (a.u.) & $a_{\perp}$ (a.u.) \\
\hline 50 & -73.66 & 47.50 \\
200 & -63.62 & 119.70 \\
800 & -78.46 & 301.62 \\
12800 & -160.84 & 1915.14 \\
204800 & -377.39 & 12160.40 \\
3276800 & -925.65 & 77213.80 \\
\hline \hline
\end{tabular}

order to probe properties of the many-body wave function. This idea was originally proposed by Kagan et al. [42], who showed that the event rate constant $K_{3}$ is proportional to the three-body local correlation function $g_{3}$. Therefore a measured reduction in $K_{3}$ from a $3 \mathrm{D}$ system to a $1 \mathrm{D}$ system in Ref. [8] was interpreted as a reduction in $g_{3}$ and a clear signature of enhanced correlations.

While a complete description of three-body recombination in atomic waveguides requires a full-scale calculation of three particles in a confinement potential, we attempt to address some of the issues within our 1D framework. Our work suggests an alternative explanation of the observed suppression of $K_{3}$ in terms of the three-body hyperradial potentials. For the experimental parameters of Ref. [8] $\left(a_{3 \mathrm{D}} \approx 100\right.$ a.u., $a_{\perp} \approx 1100$ a.u.), Olshanii's formula Eq. (61) gives a large negative $1 \mathrm{D}$ scattering length $a \approx-5300$ a.u., indicating the absence of a shallow 1D bound state. The wave function in the lowest three-body entrance channel must therefore tunnel under both the repulsive $\kappa_{\min }^{2} /\left(2 \mu R^{2}\right)$ potential in the region $R \gg|a|$ and the repulsive $\gamma_{0} /(2 \mu a R)$ (recall that $\gamma_{0}$ and $a$ are both negative) potential in the region $a_{\perp} \ll R \ll|a|$ before reaching the region $R<a_{\perp}$ where a recombination event into a deep two-body channel may occur. Therefore the measured suppression in the confined geometry could be the combined effect of the threshold law $K_{3}^{1 \mathrm{D}} \propto(\hbar / \mu)(k a)^{6}$ and the universal $R^{-1}$ barrier given in Eq. (52) leading to the suppression given in Eq. (59).

While we are confident that we have solved the 1D Schrödinger equation accurately, it is difficult to make quantitative claims regarding the measured suppression [8]. One difficulty arises from the fact that the temperature of the system in Ref. [8] is not very well characterized, and therefore it is unclear at what energy three-body collisions occur. In addition, there are a number of theoretical complications which can only be accounted for by performing a full-scale calculation of three interacting bosons in a confined geometry. For instance, since there are excited radial modes of the trap and the two-body binding energy is typically much larger than the spacing of these radial modes, there will be a series of two-body thresholds attached to each excited radial mode. All of these thresholds are open at the initial threebody threshold energy, and our calculations have not accounted for this. Also, our strictly 1D calculations undoubtedly do not correctly represent the hyperradial potentials in the region $R \leqq a_{\perp}$ where the system is neither purely 3D nor strictly $1 \mathrm{D}$ in nature. Since the potential appears in the exponent of the WKB tunneling integral, any uncertainty in the potential leads quantitatively to very different results for the suppression of $K_{3}$. Nevertheless, in view of our findings regarding universality in $1 \mathrm{D}$ systems, it is possible that the observed suppression of $K_{3}$ in Ref. [8] was a direct probe of the universal $R^{-1}$ potential Eq. (52).

We have shown in this study how inelastic processes such as three-body recombination and collision-induced breakup behave near threshold for all combinations of identical particles. For the system of three identical bosons, we have further investigated the behavior of these processes with respect to the range of the pairwise interactions and to the two-body scattering length. We have demonstrated numerically that the probability for inelastic events vanishes as the range of the interaction is taken to zero in agreement with previous analytic results [1]. Our work is related to a recent Letter that has shown how inelastic processes could in fact be possible if one considers a zero-range two-channel model with a confinement-induced Feshbach resonance [43]. Specifically, we note that finite-range interactions also break the integrability of the zero-range model by introducing energy dependence into the two-body scattering length. Finally, we have explored in detail the nature of universality for three-body systems in $1 \mathrm{D}$.

\section{ACKNOWLEDGMENTS}

We would like to thank D. Blume and B. Granger for useful discussion during the early stages of this work. N.P.M. would also like to thank M. Olshanii, Z. Walters, and S. Rittenhouse for useful discussions. We acknowledge support from the National Science Foundation.

\section{APPENDIX A: SYMMETRY OPERATIONS}

The pair permutation operators $P_{i j}$ have the following effects on the hyperangle:

$$
\begin{gathered}
P_{12} \phi=\pi-\phi, \\
P_{23} \phi=\frac{\pi}{3}-\phi, \\
P_{31} \phi=\frac{5 \pi}{3}-\phi .
\end{gathered}
$$

Solutions of definite parity may be found via the operation

$$
\Pi \phi=\pi+\phi .
$$

For three identical bosons, the symmetric projection requires

$$
\mathcal{S}=1+P_{12}+P_{23}+P_{31}+P_{12} P_{23}+P_{12} P_{31} .
$$

Similarly, for three identical fermions, the antisymmetric projection operator is

$$
\mathcal{A}=1-P_{12}-P_{23}-P_{31}+P_{12} P_{23}+P_{12} P_{31} .
$$

To determine the boundary conditions required by permutation and parity, it is useful to apply these operators to the 
TABLE III. The boundary conditions, quantization conditions, and allowed asymptotic $\kappa$ for three identical particles with squarewell interactions. The constants $\beta$ and $\phi_{b}$ are defined in Sec. III B.

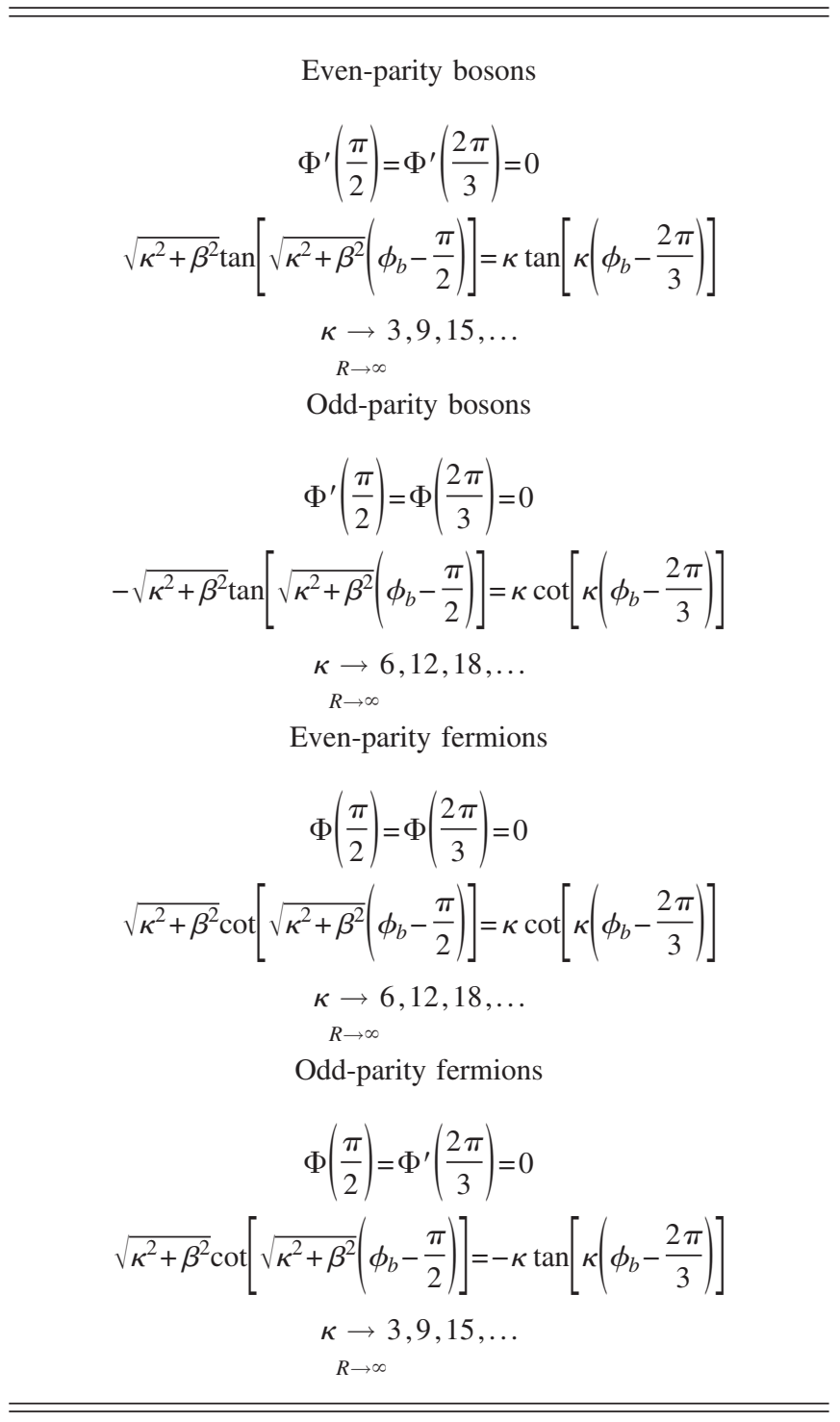

free-particle solutions of Eq. (10) [18]. Doing so, we find that the simultaneous symmetry eigenstates are real harmonics with indices that are multiples of 3. Explicitly, for bosons

$$
\Phi(\phi) \propto\left\{\begin{array}{lll}
\cos 3 n \phi, & n=0,2,4, \ldots, & \text { even parity }, \\
\sin 3 n \phi, & n=1,3,5, \ldots, & \text { odd parity }
\end{array}\right.
$$

and for fermions,

$$
\Phi(\phi) \propto\left\{\begin{array}{lll}
\sin 3 n \phi, & n=2,4,6, \ldots, & \text { even parity, } \\
\cos 3 n \phi, & n=1,3,5, \ldots, & \text { odd parity. }
\end{array}\right.
$$

From these expression, we conclude first of all that we can reduce the integration range by a factor of 12-as expected-from $2 \pi$ to only $\pi / 6$ in $\phi$. We can also conclude, for instance, that three identical fermions in the odd-parity state must obey the boundary conditions $\Phi^{\prime}(0)=0$ and $\Phi(\pi / 6)=0$.

If there are only two identical particles, then we need the operators $\mathcal{S}=1+P_{12}$ for identical bosons and $\mathcal{A}=1-P_{12}$ for identical fermions. The simultaneous symmetry eigenstates for bosons are now

$$
\Phi(\phi) \propto\left\{\begin{array}{lll}
\cos n \phi, & n=0,2,4, \ldots, & \text { even parity } \\
\sin n \phi, & n=1,3,5, \ldots, & \text { odd parity }
\end{array}\right.
$$

and for fermions

$$
\Phi(\phi) \propto\left\{\begin{array}{lll}
\sin n \phi, & n=2,4,6, \ldots, & \text { even parity, } \\
\cos n \phi, & n=1,3,5, \ldots, & \text { odd parity. }
\end{array}\right.
$$

The range of integration can in this case be reduced by a factor of 4 , and the boundary conditions extracted as above.

\section{APPENDIX B: SUMMARY OF RESULTS FOR SQUARE-WELL AND $\delta$-FUNCTION INTERACTIONS}

We summarize in this section the boundary and quantization conditions, along with the large- $R$ solutions, for all symmetries of three identical particles with square-well interactions (see Table III and Sec. III B) and for all symmetries of two identical particles with $\delta$-function interactions (see Table IV and Sec. III C).

We expect the square-well results in Table III can be generalized to arbitrary short-range interactions in basically the same way as Eq. (33). That is, the left-hand sides of the quantization conditions would get replaced by the two-body logarithmic derivative.

\section{APPENDIX C: $R$-MATRIX PROPAGATION}

In order to solve for the multichannel $S$ matrix, we use the eigenchannel R-matrix method coupled with the adiabatic hyperspherical representation. The development closely follows Refs. [44-47].

We begin with the variational expression

$$
E=\frac{\int d \phi R d R \Psi(R, \phi) H(R, \phi) \Psi(R, \phi)}{\int d \phi R d R|\Psi(R, \phi)|^{2}},
$$

assuming $\Psi$ is real without loss of generality. The Hamiltonian is given in Eqs. (8) and (9). Integrating the hyperradial kinetic energy by parts in Eq. (C1) gives the variational expression

$$
b=\frac{\int_{R_{1}}^{R_{2}} d \phi R d R\left(-\frac{\partial \Psi}{\partial R} \frac{\partial \Psi}{\partial R}+\Psi\left(k^{2}-2 \mu H_{\mathrm{ad}}\right) \Psi\right)}{\int d \phi\left[R_{2}\left|\Psi\left(R_{2}, \phi\right)\right|^{2}+R_{1}\left|\Psi\left(R_{1}, \phi\right)\right|^{2}\right]},
$$

where $b$ is minus the logarithmic derivative $[44,48]$ normal to a hypersphere, 
TABLE IV. The boundary conditions, quantization conditions, and large- $R$ solutions are shown for the two-identical-particle case. The constants $\alpha_{S}, \alpha_{D}$, and $\phi_{23}$ are defined below Eq. (34) and in Appendix B.

Even-parity bosons

$$
\begin{gathered}
\Phi^{\prime}(0)=0 \quad \text { and } \quad \Phi^{\prime}\left(\frac{\pi}{2}+\epsilon\right)=-\Phi^{\prime}\left(\frac{\pi}{2}-\epsilon\right) \\
\left(-\kappa+\frac{2}{\kappa} \mu^{2} g^{2} \alpha_{S} \alpha_{D} R^{2}\right) \tan \left[\kappa\left(\phi_{23}-\frac{\pi}{2}\right)\right]-\mu g \alpha_{S} R \tan \kappa \phi_{23} \tan \left[\kappa\left(\phi_{23}-\frac{\pi}{2}\right)\right]+\kappa \tan \kappa \phi_{23}=\mu g\left(\alpha_{S}+2 \alpha_{D}\right) R \\
\kappa=n \pi /\left|\phi_{23}-\frac{\pi}{2}\right|, \quad n=1,2,3, \ldots, \quad \text { and } \quad \kappa=(n+1 / 2) \pi /\left|\phi_{23}\right|, \quad n=0,1,2, \ldots \\
\Phi(0)=0 \quad \text { and } \quad \Phi^{\prime}\left(\frac{\pi}{2}+\epsilon\right)=-\Phi^{\prime}\left(\frac{\pi}{2}-\epsilon\right) \\
\left(-\kappa+\frac{2}{\kappa} \mu^{2} g^{2} \alpha_{S} \alpha_{D} R^{2}\right) \tan \left[\kappa\left(\phi_{23}-\frac{\pi}{2}\right)\right]-\mu g \alpha_{S} R \cot \kappa \phi_{23} \tan \left[\kappa\left(\phi_{23}-\frac{\pi}{2}\right)\right]+\kappa \cot \kappa \phi_{23}=\mu g\left(\alpha_{S}+2 \alpha_{D}\right) R \\
\kappa=n \pi /\left|\phi_{23}-\frac{\pi}{2}\right|, \quad n=1,2,3, \ldots, \quad \text { and } \quad \kappa=n \pi /\left|\phi_{23}\right|, \quad n=0,1,2, \ldots
\end{gathered}
$$

Even-parity fermions

$$
\begin{gathered}
\Phi(0)=0 \quad \text { and } \Phi\left(\frac{\pi}{2}\right)=0 \\
\kappa \cot \left[\kappa\left(\phi_{23}-\frac{\pi}{2}\right)\right]-\kappa \cot \kappa \phi_{23}=2 \mu g \alpha_{D} R \\
\kappa=n \pi /\left|\phi_{23}-\frac{\pi}{2}\right|, \quad n=1,2,3, \ldots, \quad \text { and } \quad \kappa=n \pi /\left|\phi_{23}\right|, \quad n=1,2,3, \ldots
\end{gathered}
$$

Odd-parity fermions

$$
\begin{gathered}
\Phi^{\prime}(0)=0 \quad \text { and } \quad \Phi\left(\frac{\pi}{2}\right)=0 \\
\kappa \cot \left[\kappa\left(\phi_{23}-\frac{\pi}{2}\right)\right]+\kappa \tan \kappa \phi_{23}=2 \mu g \alpha_{D} R \\
\kappa=n \pi /\left|\phi_{23}-\frac{\pi}{2}\right|, \quad n=1,2,3, \ldots, \quad \text { and } \quad \kappa=\left(n+\frac{1}{2}\right) \pi /\left|\phi_{23}\right|, \quad n=0,1,2, \ldots
\end{gathered}
$$

$$
b=-\frac{\partial \ln \Psi}{\partial \hat{n}} .
$$

In the adiabatic hyperspherical representation,

$$
\Psi(R, \phi)=\sum_{i} F_{i}(R) \Phi_{i}(R ; \phi),
$$

where $\Phi_{i}(R ; \phi)$ are eigenstates of Eq. (9) with eigenvalues $U_{i}(R)$. Substitution of the expansion Eq. (C4) into Eq. (C2) results in a set of coupled equations for $F_{i}(R)$. We further expand the radial functions in a basis set as $F_{i}(R)$ $=\sum_{n} c_{n i} B_{n}(R)$, converting the coupled equations into the generalized eigenvalue problem

$$
b \Lambda \vec{c}=\Gamma \vec{c} .
$$

The matrices $\boldsymbol{\Lambda}$ and $\boldsymbol{\Gamma}$ are defined as

$$
\Lambda_{(m, i),(n, j)}=\delta_{i, j} \sum_{n, m}\left[R_{1} B_{n}\left(R_{1}\right) B_{m}\left(R_{1}\right)+R_{2} B_{n}\left(R_{2}\right) B_{m}\left(R_{2}\right)\right],
$$

$$
\begin{aligned}
\Gamma_{(m, i),(n, j)}= & \int_{R_{1}}^{R_{2}} d R R\left[-\delta_{i, j} \frac{\partial B_{m}}{\partial R} \frac{\partial B_{n}}{\partial R}-P_{i j}\left(\frac{\partial B_{m}}{\partial R} B_{n}-B_{m} \frac{\partial B_{n}}{\partial R}\right)\right. \\
& \left.+\mathbf{P}_{i j}^{2} B_{m} B_{n}+\delta_{i, j} B_{m}\left[k^{2}-2 \mu U_{i}(R)\right] B_{n}\right] .
\end{aligned}
$$

We require the first-derivative nonadiabatic coupling,

$$
P_{i j}(R)=\left\langle\left\langle\Phi_{i}(R ; \phi)\left|\frac{\partial}{\partial R}\right| \Phi_{j}(R ; \phi)\right\rangle,\right.
$$

and the second-derivative coupling, 


$$
Q_{i j}(R)=\left\langle\left\langle\Phi_{i}(R ; \phi)\left|\frac{\partial^{2}}{\partial R^{2}}\right| \Phi_{j}(R ; \phi)\right\rangle .\right.
$$

The double angular bracket notation implies integration only over $\phi$. The square of $\mathbf{P}$ is given by the symmetric part of $\mathbf{Q}$ through the relation $Q_{i j}=\mathbf{P}_{i j}^{2}+\partial P_{i j} / \partial R$ :

$$
\mathbf{P}_{i j}^{2}=-\left\langle\left\langle\frac{\partial \Phi_{i}(R, \phi)}{\partial R} \mid \frac{\partial \Phi_{j}(R ; \phi)}{\partial R}\right\rangle .\right.
$$

Equation (C5) is solved for the expansion coefficients $\vec{c}$, yielding a solution in the region $R_{1} \leqslant R \leqslant R_{2}$. For the first region $0 \leqslant R \leqslant R_{1}$, we require $F_{i}^{\prime}(0)=0$ but impose no boundary condition at $R_{1}$. The generalized eigenvalue problem then yields an eigenvalue $b_{\beta}$ and wave function $F_{i \beta}$ for each open or weakly closed channel at $R_{1}$.

To propagate the solution to large $R$, we solve the system of equations again in the region $R_{1} \leqslant R \leqslant R_{2}$ with no boundary conditions at either $R_{1}$ or $R_{2}$.

A valid solution in the region $0 \leqslant R \leqslant R_{2}$ is then constructed by matching the solutions from the two regions at $R_{1}$ and by requiring that the overall solution be an eigenchannel solution of the $\mathbf{R}$ matrix,

$$
\mathbf{R}=(\mathbf{F})\left(\mathbf{F}^{\prime}\right)^{-1} .
$$

That is, $\Psi$ should have a constant normal derivative at the surface $R=R_{2}$. This procedure is repeated until the solutions can be accurately matched to analytic asymptotic forms [45-47].

To be more explicit, we write the full wave function outside the $\mathbf{R}$-matrix volume as

$$
\Psi_{\beta}^{\text {out }}(R, \phi)=\sum_{j} \Phi_{j}(R ; \phi)\left[f_{j}(R) I_{j \beta}-g_{j}(R) J_{j \beta}\right],
$$

where $f_{j}(R)$ and $g_{j}(R)$ are

$$
\begin{aligned}
& f_{j}(R)= \begin{cases}\sqrt{\frac{2}{\pi k_{f} R}} \cos k_{f} R & \text { if } j \text { is a two-body channel, } \\
J_{\kappa}(k R) & \text { if } j \text { is a three-body channel, }\end{cases} \\
& g_{j}(R)= \begin{cases}\sqrt{\frac{2}{\pi k_{f} R}} \sin k_{f} R & \text { if } j \text { is a two-body channel, } \\
Y_{\kappa}(k R) & \text { if } j \text { is a three-body channel. }\end{cases}
\end{aligned}
$$

The order $\kappa$ is determined as described in Sec. III. The solution inside the volume involves the numerical functions $F_{j \beta}$,

$$
\Psi_{\beta}^{\mathrm{in}}(R ; \phi)=\sum_{j} F_{j \beta}(R) \Phi_{j}(R ; \phi) .
$$

The matrices $\mathbf{I}$ and $\mathbf{J}$ are obtained from matching at some large distance $R=R_{M}$, which is conveniently accomplished using

$$
I_{j \beta}=W\left(g_{j}, F_{j \beta}\right) / W\left(g_{j}, f_{j}\right)
$$

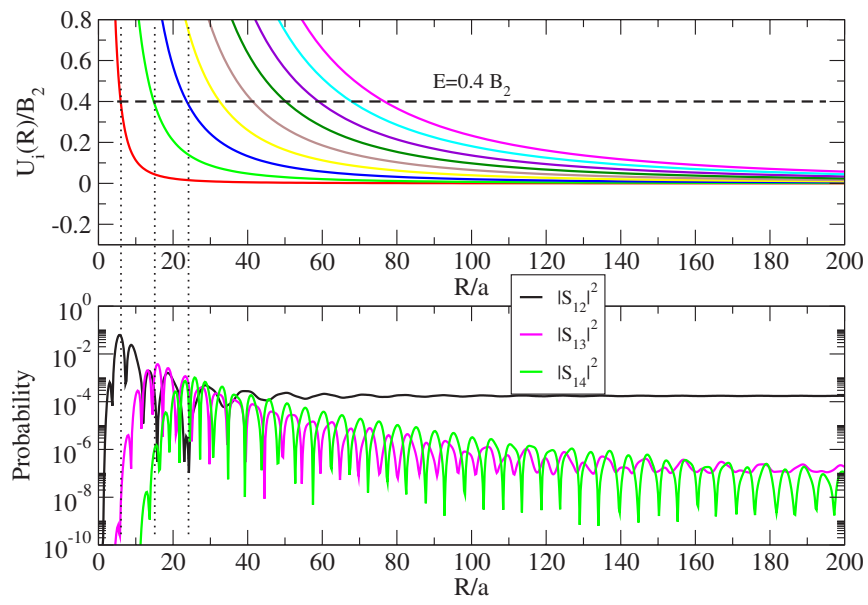

FIG. 7. (Color online) (a) Three-body potential curves for the Pöschl-Teller two-body potential with $L=2$ and $D=1 / 2$. The horizontal dashed line indicates the collision energy, and the vertical dotted lines mark the classical turning points. (b) The convergence of the transition probability between the two-body channel and the lowest three three-body channels at $E=0.4 B_{2}$ as a function of the matching distance.

$$
J_{j \beta}=W\left(f_{j}, F_{j \beta}\right) / W\left(g_{j}, f_{j}\right),
$$

where $W(f, g)$ denotes the Wronskian of $f$ and $g$. Defining $(\mathbf{f})_{i j}=\delta_{i j} f_{i}$ and $(\mathbf{g})_{i j}=\delta_{i j} g_{i}$, the $K$ matrix is

$$
\mathbf{K}\left(R_{M}\right)=\left(\mathbf{f}-\mathbf{f}^{\prime} \mathbf{R}\right)\left(\mathbf{g}-\mathbf{g}^{\prime} \mathbf{R}\right)^{-1} .
$$

The $\mathbf{R}$ matrix is calculated via Eq. (C10) using the numerical solutions $F_{i \beta}$ and the result $F_{i \beta}^{\prime}\left(R_{M}\right)=-b_{\beta} F_{i \beta}\left(R_{M}\right)$. The latter holds only at large $R$ where $P_{i j} \rightarrow 0$, since the exact relation is

$$
-b_{\beta} F_{i \beta}=F_{i \beta}^{\prime}+\sum_{j} P_{i j} F_{j \beta},
$$

in which each quantity is evaluated at $R_{M}$. This relation is obtained by differentiating Eq. (C13) and projecting the result onto $\Phi_{i}$. Finally, the $S$ matrix is calculated from $\mathbf{K}$ using

$$
\mathbf{S}\left(R_{M}\right)=\frac{1+i \mathbf{K}\left(R_{M}\right)}{1-i \mathbf{K}\left(R_{M}\right)} .
$$

It is important to propagate the $\mathbf{R}$ matrix to large $R$ in order to obtain a converged, unitary $S$ matrix. In Fig. 7, we show the convergence of a few $S$-matrix elements with the matching distance $R_{M}$ for an eight-channel calculation using the Pöschl-Teller potential Eq. (47) with $L=2$ and $D=1 / 2$. Figure 7(a) shows the lowest nine potential curves corresponding to three-body channels. (Since it converges to -1 on the scale of the figure, the two-body channel is not visible.) The horizontal dashed line shows the energy at which the calculation in Fig. 7(b) was carried out, and the vertical dotted lines mark the classical turning points for the first three channels. Note that the probability $\left|S_{1 i}\left(R_{M}\right)\right|^{2}$ peaks approximately at the classical turning point for $U_{i}(R)$.

For the calculations presented in Fig. 2, we propagated the $\mathbf{R}$ matrix to $R=1000 a$ to assure convergence. It is evident 
from Fig. 7 that the quantity $\left|S_{12}\right|^{2}$ has already converged by $R=200 a$. We have also verified that this probability is stable with respect to the inclusion of more coupled channels. The other probabilities, however, have not yet converged as well, although their magnitude makes them negligible.

Our calculation of the matrix elements in Eq. (C6) is simplified by using $B$ splines as our radial basis set $\left\{B_{n}(R)\right\}[49]$.
This choice also simplifies the imposition of boundary conditions since $B$ splines have only local support. We typically use ten fifth-order $B$ splines within each $\mathbf{R}$-matrix sector, leading to a $(10 \times N) \times(10 \times N)$ matrix equation $(N$ is the number of channels). The size of the sectors $R_{i+1}-R_{i}$ is chosen to be no more than one de Broglie wavelength in the lowest (two-body) channel.
[1] J. B. McGuire, J. Math. Phys. 5, 622 (1964).

[2] C. N. Yang, Phys. Rev. Lett. 19, 1312 (1967).

[3] E. Lieb and W. Liniger, Phys. Rev. 130, 1605 (1963).

[4] H. B. Thacker, Phys. Rev. D 11, 838 (1974).

[5] M. D. Girardeau, Phys. Rev. 139, B500 (1965).

[6] L. Tonks, Phys. Rev. 50, 955 (1936).

[7] A. Görlitz et al., Phys. Rev. Lett. 87, 130402 (2001).

[8] B. Laburthe Tolra, K. M. O’Hara, J. H. Huckans, W. D. Phillips, S. L. Rolston, and J. V. Porto, Phys. Rev. Lett. 92, 190401 (2004).

[9] T. Kinoshita, T. Wenger, and D. S. Weiss, Nature (London) 440, 900 (2006).

[10] T. Kinoshita, T. Wenger, and D. S. Weiss, Science 305, 1125 (2004)

[11] T. P. Meyrath, F. Schreck, J. L. Hanssen, C. S. Chuu, and M. G. Raizen, Phys. Rev. A 71, 041604(R) (2005).

[12] J. Esteve, J. B. Trebbia, T. Schumm, A. Aspect, C. I. Westbrook, and I. Bouchoule, Phys. Rev. Lett. 96, 130403 (2006).

[13] M. Olshanii, Phys. Rev. Lett. 81, 938 (1998).

[14] E. Braaten and H. Hammer, Phys. Rep. 428, 259 (2006).

[15] E. Nielsen and J. H. Macek, Phys. Rev. Lett. 83, 1566 (1999).

[16] E. Nielsen, D. V. Fedorov, A. S. Jensen, and E. Garrido, Phys. Rep. 347, 373 (2001).

[17] B. D. Esry, C. H. Greene, and J. P. Burke, Jr., Phys. Rev. Lett. 83, 1751 (1999).

[18] H. Suno, B. D. Esry, C. H. Greene, and J. P. Burke, Jr., Phys. Rev. A 65, 042725 (2002).

[19] J. P. D’Incao and B. D. Esry, Phys. Rev. A 72, 032710 (2005).

[20] E. Wigner, Phys. Rev. 73, 1002 (1948).

[21] H. R. Sadeghpour, J. L. Bohn, M. J. Cavegnero, B. D. Esry, I. I. Fabrikant, J. H. Macek, and A. R. P. Rau, J. Phys. B 33, R93 (2000)

[22] B. D. Esry, C. H. Greene, and H. Suno, Phys. Rev. A 65, 010705(R) (2001).

[23] C. D. Lin, Phys. Rep. 257, 1 (1995).

[24] J. H. Macek, J. Phys. B 1, 831 (1968).

[25] W. G. Gibson, S. Y. Larsen, and J. Popiel, Phys. Rev. A 35, 4919 (1987).

[26] N. P. Mehta and J. R. Shepard, Phys. Rev. A 72, 032728 (2005).

[27] O. Chuluunbaatar, A. A. Gusev, M. S. Kaschiev, V. A. Kaschieva, A. Amaya-Tapia, S. Y. Larsen, and S. I. Vinitsky, J. Phys. B 39, 243 (2006).
[28] O. Chuluunbaatar, A. A. Gusev, S. Y. Larsen, and S. I. Vinitsky, J. Phys. A 35, L513 (2002).

[29] C. Felline, N. P. Mehta, J. Piekarewicz, and J. R. Shepard, Phys. Rev. C 68, 034003 (2003).

[30] B. D. Esry, H. Suno, and C. H. Greene, in The Expanding Frontier of Atomic Physics, Proceedings of the XVIII International Conference on Atomic Physics (ICAP 2002), edited by H. R. Sadeghpour, E. J. Heller, and D. E. Pritchard (World Scientific, Singapore, 2003).

[31] P. M. Morse and H. A. Feshbach, Methods of Theoretical Physics, 1st ed. (McGraw-Hill, New York, 1953).

[32] C. H. Greene, J. P. Burke, Jr., and B. D. Esry (unpublished).

[33] A. Amaya-Tapia, S. Y. Larsen, and J. Popiel, Few-Body Syst. 23, 87 (1997).

[34] A. G. Abrashkevich, M. S. Kaschiev, and S. I. Vinitsky, J. Comput. Phys. 163, 328 (2000).

[35] M. G. Dimova, M. S. Kaschiev, and S. I. Vinitsky, J. Phys. B 38, 2337 (2005).

[36] O. I. Kartavtsev and A. V. Malykh, Phys. Rev. A 74, 042506 (2006).

[37] L. D. Landau and E. M. Lifshitz, Course of Theoretical Physics, Vol. 3: Quantum Mechanics: Non-Relativistic Theory (Pergamon Press, Oxford, 1958).

[38] G. P. Lepage, From Actions to Answers (TASI-89) (World Scientific, Singapore, 1989); e-print arXiv:nucl-th/9706029.

[39] A. R. Janzen and A. R. Aziz, J. Chem. Phys. 103, 9626 (1995).

[40] V. Efimov, Sov. J. Nucl. Phys. 10, 62 (1970).

[41] J. P. D'Incao and B. D. Esry, Phys. Rev. Lett. 94, 213201 (2005).

[42] Y. Kagan, B. V. Svistunov, and G. V. Shlyapnikov, JETP Lett. 42, 209 (1985).

[43] V. A. Yurovsky, A. Ben-Reuven, and M. Olshanii, Phys. Rev. Lett. 96, 163201 (2006).

[44] M. Aymar, C. H. Greene, and E. Luc-Koenig, Rev. Mod. Phys. 68, 1015 (1996).

[45] J. P. Burke, Jr., Ph.D. thesis, University of Colorado, 1999.

[46] O. I. Tolstikhin and H. Nakamura, J. Chem. Phys. 108, 8899 (1998).

[47] K. L. Baluja, P. G. Burke, and L. A. Morgan, Comput. Phys. Commun. 27, 299 (1982).

[48] C. H. Greene, Phys. Rev. A 28, 2209 (1983).

[49] C. de Boor, A Practical Guide to Splines (Springer, Berlin, 1978). 